\title{
Erratum: Bird, Quinton, and Cumming (2021)
}

In the article Bird, G.A., Quinton, M.L., \& Cumming, J. (2021). Promoting athlete mental health: The role of emotion regulation. Journal of Clinical Sport Psychology, Advanced online publication, https://doi.org/10.1123/jcsp.2021-0022, there was an error in the reporting of the regressional analyses. Some of the beta values reported in the text did not match the values in the table. The online version of this article has been corrected. The authors apologize for this error. 